\title{
On the possible existence of a self-regulating hydrodynamical process in slowly rotating stars
}

\section{Lithium plateau in halo stars and primordial abundance}

\author{
S. Théado and S. Vauclair \\ Laboratoire d'Astrophysique, 14 Av. E. Belin, 31400 Toulouse, France
}

Received 26 December 2000 / Accepted 25 May 2001

\begin{abstract}
The lithium plateau observed in halo stars has long appeared as a paradox in the general context of the lithium abundance behavior in stellar outer layers. First, the plateau is flat, second, the lithium abundance dispersion is extremely small. This seems in contradiction with the large lithium variations observed in younger stars. It is also difficult to understand theoretically: as lithium nuclei are destroyed by nuclear reactions at a relatively low temperature $(\cong 2.5$ million degrees), the occurrence of macroscopic motions in the stellar outer layers easily lead to lithium depletion at the surface. On the other hand, if no macroscopic motions occur in the stellar gas, lithium is subject to microscopic diffusion which, in the case of halo stars, should also lead to depletion. Several ideas have been proposed to account for the lithium behavior in halo stars. The most promising possibilities were rotational-induced mixing, which could reduce lithium in the same way for all the stars (Vauclair 1988; Pinsonneault et al. 1992 and 1999) and mass-loss, which could oppose the lithium settling (Vauclair \& Charbonnel 1995, 1998). In both cases however, the parameters should be tightly adjusted to prevent any dispersion in the final results. Vauclair (1999) (Paper I) looked for a physical process which could occur in slowly rotating stars and explain why the dispersion of the lithium abundances in the halo stars' plateau is so small. She pointed out that the $\mu$-gradient terms which appear in the computations of the meridional circulation velocity (e.g. Mestel 1953) were not introduced in previous computations of rotationally-induced mixing. This can lead to a self-regulating process which reduces the efficiency of the meridional circulation as well as the microscopic diffusion. Here we present numerical computations of this process and its influence on the lithium abundance variations in halo stars. We show that in slowly rotating stars, under some conditions, lithium can be depleted by a factor of up to two with a dispersion smaller than 0.1 dex in the middle part of the lithium plateau. We derive a primordial lithium abundance of $2.5 \pm 0.1$, consistent with the recent determinations of $\mathrm{D} / \mathrm{H}$ and ${ }^{4} \mathrm{He} / \mathrm{H}$.
\end{abstract}

Key words. diffusion - hydrodynamics - stars: abundances - stars: population II

\section{Introduction}

Prior to the first lithium detection in halo stars by Spite \& Spite (1982), the primordial ${ }^{7} \mathrm{Li}$ value was believed to lie around $A(\mathrm{Li})=3.3$ (in the $\log H=12$ scale), which corresponds to both the meteoritic and the maximum lithium abundance in Pop I stars throughout the entire observed $T_{\text {eff }}$ range $(8000-4500 \mathrm{~K})$. Below this maximum value, the observations revealed a large scatter in the $\mathrm{Li}-T_{\text {eff }}$ plane, with an evidence of lithium decrease with age for stars of similar $T_{\text {eff }}$.

For this reason it was expected that, due to their age, halo stars should have destroyed all their lithium. In this

Send offprint requests to: S. Théado,

e-mail: stheado@obs-mip.fr context, the first lithium detection in these stars, with a value $A(\mathrm{Li})=2.1$, only one order of magnitude smaller than the maximum Pop I value, was a surprise. The shock was still stronger when the observations in different stars, with different masses and metallicities, presented the same lithium value over a range of $800 \mathrm{~K}$ in effective temperature $(6250-5480 \mathrm{~K})$.

From then on, the observers switched to another extreme: the idea that lithium should be depleted in these stars was completely rejected on the basis of the constancy and very small dispersion of its abundances. The observed value was then supposed to represent the primordial abundance, without any modification, and was "sold" as such to cosmologists. Meanwhile, theoreticians went on claiming that, from all possible computations of stellar 
structure, the lithium abundance could not keep exactly the same value in stars during 14 billions years, without any alteration.

In the present paper, we argue that we can reconcile the theory with the observations and obtain a consistent view of the lithium behavior in stars.

In Sect. 2, we discuss recent improvements on the observational side and show that the careful studies of the lithium abundances in the "Spite plateau", obtained from various authors, lead to the conclusion that the dispersion is smaller than 0.1 dex. Meanwhile, the detection of ${ }^{6} \mathrm{Li}$, if confirmed, puts strong constraints on the possible lithium destruction by nuclear reactions in these stars (otherwise no ${ }^{6} \mathrm{Li}$ should be left).

A theoretical discussion about previous models for lithium abundance variations in population II stars is given in Sect. 3.

In Sect. 4, we show how the introduction of the effect of $\mu$-gradients in the computations of rotation-induced mixing, as discussed by Vauclair (1999) (Paper I) modifies the previously published results. We argue that a self-regulating process may take place, which may explain most of the lithium observations in halo stars.

Section 5 is devoted to the results obtained from numerical computations of this process and its influence on the lithium abundance variations in different stars with various masses, rotational velocities and metallicities.

We give our conclusions in Sect. 6, including a discussion about the assumptions introduced in our computations. In the framework of our model, we obtain a primordial lithium abundance of $2.5 \pm 0.1$.

\section{The lithium plateau: Observational constraints}

Since the first observations of the lithium plateau by Spite \& Spite (1982), many abundance determinations have confirmed the constancy and very small dispersion of the lithium value in most halo stars with effective temperatures larger than $5500 \mathrm{~K}$. This result lead the observers to the conclusion that the observed lithium abundance was exactly the pristine value, although theoretical computations predicted some lithium depletion in these stars. We first give a short summary of the important observational constraints and then we will discuss the theoretical models proposed in the literature.

\subsection{Trends with temperature and metallicity}

The observed lithium plateau is flat, at least to a first approximation. Some doubts still appear, however, about possible small variations with effective temperature and/or metallicity.

Thorburn (1994) found that the lithium plateau presented a positive slope with $T_{\text {eff }}$ which, she argued, would not exist if the lithium was primordial. She also found a general trend of the lithium abundance with metallicity which could explain part of the scatter that she observed on the "Spite plateau". She suggested that the slight increase of the lithium abundance with $[\mathrm{Fe} / \mathrm{H}]$ could be due to a small production of lithium in the early life of the galaxy.

While Norris et al. (1994) and Ryan et al. (1996) confirmed these results, Molaro et al. (1995) argued that the slope of the plateau disappears when a subset of stars with temperature based on Balmer lines profiles is adopted. They found no correlation between lithium abundances and metallicity for $[\mathrm{Fe} / \mathrm{H}]<-1.4$.

Later on, Bonifacio \& Molaro (1997) studied a sample of 41 plateau stars, to investigate in more detail the distribution of lithium abundances with $[\mathrm{Fe} / \mathrm{H}]$ and $T_{\text {eff }}$. They used a new effective temperature scale based on the new accurate infrared flux method (IRFM, Blackwell et al. 1990) applied to a large sample of stars by Alonso et al. (1996). They found only a tiny trend with $T_{\text {eff }}$ and no trend with $[\mathrm{Fe} / \mathrm{H}]$. Using mean lithium values corrected for standard depletion and NLTE effects, they give for the primordial abundance: $A\left(\mathrm{Li}_{0}\right)=2.238 \pm 0.012$.

Meanwhile Ryan et al. (1999) studied 23 very metalpoor stars chosen to enable a precise measurement of the dispersion in the "Spite plateau": they found no evidence for a trend with $T_{\text {eff }}$ but they do recover a strong dependence of the lithium abundance with metallicity, which they attribute to the chemical evolution in the early galaxy. The value they derive for the primordial abundance, taking into account galactic chemical evolution but no depletion, lies below the mean plateau value: 2.0 dex only, with a systematic error of 0.1 dex.

While the question of slight lithium slopes with effective temperature and/or metallicity is not yet completely settled, everyone agrees on the fact that some stars otherwise indistinguishable from normal plateau stars show large lithium deficiencies, well below the plateau itself (Hobbs et al. 1991; Thorburn 1994; Norris et al. 1997). Although these stars are certainly lithium depleted, so that their observed abundances are quite different from the pristine one, they must be accounted for in the theoretical scenarios. Norris et al. (1997) measured the abundances of 14 elements (Fe, Mg, Al, Si, Ca, Sc, Ti, Cr, Mn, $\mathrm{Co}, \mathrm{Ni}, \mathrm{Sr}, \mathrm{Y}, \mathrm{Ba})$ for the metal poor stars G66-30, G139-8 and G186-26, which are highly lithium deficient and lie near the main sequence cut-off. They found no abundance anomalies for the other elements that one might associate with the lithium deficiencies. This result is in favor of a nuclear lithium destruction and negligible element settling in these stars.

\subsection{Lithium abundance dispersion in the plateau}

Since 1982, many authors have discussed the possible existence of an intrinsic dispersion in the "Spite plateau". Deliyannis et al. (1993), supported by Thorburn (1994), 
argued that the spread in measured plateau stars abundances exceeded that expected from observational data. They gave values of about 20\% (Deliyannis et al. 1993) to $25 \%$ (Thorburn 1994). Molaro et al. (1995), Spite et al. (1996) and Bonifacio \& Molaro (1997) have questioned whether some of the error estimates in these earlier works were realistic and have suggested that the dispersion is smaller. According to Molaro et al. (1995), all the observations are consistent with the same pristine lithium abundance and the errors estimated for individual stars account for the observed dispersion. Spite et al. (1996) argued that the scatter is fully explained by the temperature equivalent width errors and that the intrinsic scatter, if real, is small. Bonifacio \& Molaro (1997) revised the lithium abundances in a selected sample of halo stars using the infrared flux method (IRFM) temperatures by Alonso et al. (1996). They found no evidence for intrinsic dispersion.

In the sample of Ryan et al. (1999), 21 stars out of 23 have abundances consistent with an observed spread of a mere 0.031 dex with reference to the metallicity trend that they claim to observe. Because the formal errors are 0.033 dex, they conclude that the intrinsic spread of the $\mathrm{Li}$ abundances at a given metallicity is lower than 0.02 dex and consistent with zero at the very metal poor halo turnoff. This maximum dispersion is much lower than those obtained by previous studies (Spite et al. $\simeq 0.06-$ 0.08 dex, Bonifacio \& Molaro 0.07 dex) who did not introduce any systematic variation with metallicity.

The situation is different in globular clusters where the lithium abundances in stars around the turn-off seem to show a dispersion larger than that observed for field stars. Deliyannis et al. (1995) and Boesgaard et al. (1998) observed the stars near the turnoff of the old metal poor globular cluster M92. They reveal a spread in abundances of a factor of 2-3 for a small sample of stars. Moreover some of them show a high lithium abundance compared to the halo field plateau.

These variations inside globular clusters may be related to local pollution and other effects which will not be discussed here. In any case the very small lithium dispersion in halo field stars (probably less than 10\% from the most recent studies), acts as a strong constraint on the theoretical models.

As we will see below, these results argue against strong lithium depletion in plateau stars. Recent observations of ${ }^{6} \mathrm{Li}$ confirm this idea. ${ }^{6} \mathrm{Li}$ has indeed been observed in the atmosphere of the halo stars HD 84937 by Smith et al. (1993), Hobbs \& Thorburn (1994), Hobbs \& Thorburn (1997) and $\mathrm{BD}+26^{\circ} 3578$ by Smith et al. (1998). As this light lithium isotope is more easily destroyed than ${ }^{7} \mathrm{Li}$, these observations, if confirmed, seem to prove that no strong lithium destruction by nuclear reactions occured in these stars during main sequence or pre-main sequence. Small destruction rates may however be allowed: we will see that a ${ }^{7} \mathrm{Li}$ depletion by a factor of two leads to a ${ }^{6} \mathrm{Li}$ decrease by less than a factor of five.

\section{The lithium plateau: Theoretical discussion}

The expected lithium depletion in halo stars is primarily due to the diffusion processes which take place in the radiative regions inside the stars, below the outer convective zones. Due to pressure and temperature gradients, lithium settles down, as well as helium and other heavier elements. Mixing induced by rotation, internal waves, or mass loss related motions may slow down the settling process, but then it brings up to the convective zone matter in which lithium has been destroyed by nuclear reactions.

These lithium depleting processes have been extensively studied in the literature. Michaud et al. (1984) first computed the lithium abundance variations in halo stars including element separation. Their models predicted a downward curvature in the ${ }^{7} \mathrm{Li}$ isochrones for increasing effective temperature, in contradiction with the observations. This result, simply due to the decrease of the convective depth for hotter stars, has been confirmed many times in the literature (Deliyannis et al. 1990; Deliyannis \& Demarque 1991; Proffitt \& Michaud 1991; Chaboyer \& Demarque 1994; Vauclair \& Charbonnel 1995).

Models including rotation-induced mixing were proposed to prevent the effect of element separation. As such a mixing leads to lithium destruction by nuclear reactions, it was suggested that this destruction could be identical for all the stars, so that the lithium plateau could be preserved (Vauclair 1988; Pinsonneault et al. 1992, 1999; Chaboyer \& Demarque 1994). However, if a constant lithium abundance can be obtained in this case, it becomes extremely difficult to account for such a small dispersion as observed.

Stellar models which include instabilities related to angular momentum and chemical species transport require additional input physics beyond standard models. The important new ingredients include:

1- a distribution of initial angular momenta;

2- a description for angular momentum losses;

3- a prescription for the internal transport of angular momentum and for the associated mixing processes in the stellar radiative regions.

Using various initial angular momenta, Pinsonneault et al. (1992) obtained lithium depletion factors between 5 and 10 at fixed $T_{\text {eff }}$. The lithium abundances in the plateau slightly decreased for larger $T_{\text {eff }}$ in contradiction with the observations (this trend was already predicted by Vauclair 1988). This effect however is quite attenuated for older ages (14 billion years) as the more lithium depleted stars at the hot end of the plateau move into the giant branch.

Chaboyer \& Demarque (1994) could also reproduce a lithium plateau with a large lithium depletion in a similar framework, using different angular momentum loss and rotational mixing laws. They included meridional circulation, dynamical shear, secular shear, Solberg-Hoiland and Goldreich-Schubert-Fricke instabilities. Their results also showed the same trend of abundance decrease with increasing $T_{\text {eff }}$. 
Using a distribution of initial angular momenta as inferred from stellar rotation data in young open clusters, Pinsonneault et al. (1999) obtained a well defined, nearly flat lithium plateau, without the downward trend at the hot end. A modest scatter does remain however, which increases with the average lithium depletion: this constraint excludes a lithium depletion larger than a factor of 3 .

Considering the difficulty for theoretical computations, including element settling and rotation induced mixing, to account for both the flatness of the lithium plateau and the small dispersion, Vauclair \& Charbonnel (1995) and Vauclair \& Charbonnel (1998) suggested taking into account a stellar wind. In some cases, a wind could prevent the element settling without bringing up to the convective zone lithium depleted matter. Computations were done for various mass loss rates, between 1 and 1000 times the solar wind. Rates larger than 10 times the solar wind were needed to have any effect on the lithium depletion, and the best results were obtained for rates of about $10^{-12} M_{\odot} \mathrm{yr}^{-1}$. In this case the lithium plateau was nicely reproduced. For larger rates, nuclearly depleted matter began to migrate up into the outer layers, rapidly leading to large lithium destruction, in contradiction with the observations. Although appealing, this model suffers from the fact that it needs winds larger than the solar wind, and that various rates lead to a non-negligible scatter in the results.

The fact that the dispersion in the lithium plateau is extremely small compared to the observational errors is the strongest constraint for the theoretical models. Several assumptions have lead to models which could account for the lithium abundance constancy, but the small observed scatter could only be reproduced using some "ad hoc" hypothesis on the initial parameters.

In all these computations, however, the effect of the $\mu$-gradients induced by element separation was not taken into account. In the present paper, we claim that when it is introduced in the computations of rotation-induced mixing, this process can explain the features observed in halo stars, including the very small dispersion.

\section{Diffusion and mixing in the presence of $\mu$-gradients}

The meridional circulation velocity in stars, in the presence of $\mu$-gradients, is the sum of two terms, one due to the classical thermal imbalance ( $\Omega$-currents) and the other one due to the induced horizontal $\mu$-gradients ( $\mu$-induced currents, or $\mu$-currents in short). In the most general cases, $\mu$-currents are opposite to $\Omega$-currents (Mestel 1953; Zahn 1992; Maeder \& Zahn 1998). When element settling occurs below the stellar outer convective zone in cool stars, a small helium gradient builds, even in the presence of circulation.

Then a new process must take place, which had not been taken into account in previous computations of diffusion-induced $\mu$-gradients. Mestel \& Moss (1986) gave a lengthy discussion of this effect for nuclearly-induced $\mu$-gradients. Chaboyer et al. (1995) simulated a $\mu$-gradient effect in their diffusion computations in the form of a reducing factor $f_{\mu}$ which was taken as a parameter. Vauclair (1999) (Paper I) showed that the resulting $\mu$-gradients are rapidly large enough to create $\mu$-currents of the same order as $\Omega$-currents. Then a self-regulating process may take place, in which both the circulation and the settling are strongly reduced.

Here we give precise computations of the $\mu$-currents with the following simplifying assumptions: differential rotation is supposed negligible inside the stars (as it is presently in the sun) and the stellar rotation velocity is taken as constant along the stellar lifetime. These assumptions neglect the possibility of rotational breaking in early stages of stellar evolution. This is discussed in the conclusion.

\subsection{Computations of $\Omega$ and $\mu$-currents}

In the present paper, we have computed these currents in halo stars for different metallicities and rotation velocities and we have done a complete treatment of the resulting diffusion of the chemical species, including mixing and settling.

As in Paper I, we have neglected the deviations from a perfect gas law as well as the energy production terms, which are completely negligible in the regions of the star where the process takes place.

We also assumed a negligible differential rotation, as observed inside the Sun from helioseismic studies. The corresponding condition on $\Omega$ is:

$\left|\frac{\partial \ln \Omega}{\partial \ln r}\right|<\frac{\Omega^{2} r^{3}}{G M}$.

The vertical meridional circulation velocity may be written (Paper I):

$u_{\mathrm{r}}=\frac{\nabla_{\mathrm{ad}}}{\nabla_{\mathrm{ad}}-\nabla+\nabla_{\mu}} \frac{\varepsilon_{\Omega}}{g}$

where $g$ represents the local gravity, $\nabla_{\text {ad }}$ and $\nabla$ the usual adiabatic and real ratios $\left(\frac{\mathrm{d} \ln T}{\mathrm{~d} \ln P}\right)$ and $\nabla_{\mu}$ the mean molecular weight contribution $\left(\frac{\mathrm{d} \ln \mu}{\mathrm{d} \ln P}\right)$.

The expression of $\varepsilon_{\Omega}$ is obtained as a function of the $\Omega$ and $\mu$-currents:

$\varepsilon_{\Omega}=\left(\frac{L}{M}\right)\left(E_{\Omega}+E_{\mu}\right) P_{2}(\cos \theta)$

with:

$$
\begin{aligned}
E_{\Omega}= & \frac{8}{3}\left(\frac{\Omega^{2} r^{3}}{G M}\right)\left(1-\frac{\Omega^{2}}{2 \pi G \bar{\rho}}\right) \\
E_{\mu}= & \frac{\rho_{\mathrm{m}}}{\bar{\rho}}\left\{\frac{r}{3} \frac{\mathrm{d}}{\mathrm{d} r}\left[\left(H_{\mathrm{T}} \frac{\mathrm{d} \Lambda}{\mathrm{d} r}\right)-\left(\chi_{\mu}+\chi_{\mathrm{T}}+1\right) \Lambda\right]\right. \\
& \left.-\frac{2 H_{\mathrm{T}} \Lambda}{r}\right\}
\end{aligned}
$$

Here $\bar{\rho}$ represents the density average on the level surface $(\simeq \rho)$ while $\rho_{\mathrm{m}}$ is the mean density inside the sphere of 
radius $r ; H_{\mathrm{T}}$ is the temperature scale height; $\Lambda$ represents the horizontal $\mu$ fluctuations $\frac{\tilde{\mu}}{\bar{\mu}} ; \chi_{\mu}$ and $\chi_{\mathrm{T}}$ represent the derivatives:

$\chi_{\mu}=\left(\frac{\partial \ln \chi}{\partial \ln \mu}\right)_{\mathrm{P}, \mathrm{T}} ; \quad \chi_{\mathrm{T}}=\left(\frac{\partial \ln \chi}{\partial \ln T}\right)_{\mathrm{P}, \mu}$.

While the term with the second derivative of $\Lambda$ was neglected in Paper I, these expressions are extensively computed in the present numerical computations.

Writing $u_{\mathrm{r}}$ as:

$u_{\mathrm{r}}=U_{\mathrm{r}} P_{2}(\cos \theta)$

the horizontal meridional velocity is given by:

$u_{\theta}=-\frac{1}{2 \rho r} \frac{\mathrm{d}}{\mathrm{d} r}\left(\rho r^{2} U_{\mathrm{r}}\right) \sin \theta \cos \theta$.

In the following, we also use the notations $U_{\Omega}$ and $U_{\mu}$ defined as:

$U_{\mathrm{r}}=U_{\Omega}+U_{\mu}$

where $U_{\Omega}$ represents the part of $U_{\mathrm{r}}$ which includes $E_{\Omega}$, and $U_{\mu}$ the part including $E_{\mu}$, namely:

$U_{\Omega}=\frac{\nabla_{\mathrm{ad}}}{\nabla_{\mathrm{ad}}-\nabla+\nabla_{\mu}} \frac{E_{\Omega}}{g} \frac{L}{M}$

idem for $U_{\mu}$.

The expressions and orders of magnitude of the horizontal $\mu$-gradients $\Lambda$ were extensively discussed in Paper I. In all cases, on a given level surface $\Lambda$ is proportional to the vertical $\mu$-gradient. A general expression can be given in the form:

$\Lambda=\frac{\tilde{\mu}}{\mu}=\alpha_{\Lambda} \cdot r \cdot \nabla \ln \mu$

where $\alpha_{\Lambda}$ is a constant, which is related to the horizontal diffusion coefficient in case of shear flow instabilities by (Zahn 1993, Paper I):

$\alpha_{\Lambda}=\frac{1}{6 \alpha_{\mathrm{h}}} \quad$ where $\quad \alpha_{\mathrm{h}}=\frac{D_{\mathrm{h}}}{\left(U_{\mathrm{r}} \cdot r\right)}$.

\subsection{Element diffusion and abundance variations}

As discussed many times in the literature, stellar regions in which no macroscopic motions take place are subject to element settling induced by pressure and temperature gradients and radiative acceleration (Michaud 1970; Michaud et al. 1976 (MCV2); Vauclair \& Vauclair 1982; Vauclair \& Charbonnel 1995, 1998). When macroscopic motions occur, they slow down the settling but do not prevent it completely until an equilibrium concentration gradient is reached (Vauclair et al. 1978a (V2M), 1978b (V2SM); Richard et al. 1996).

In Paper I we showed that in halo stars this equilibrium gradient is never reached before the $\mu$-currents become of the same order of magnitude as the $\Omega$-currents. Thus the abundance variations induced by settling and rotationinduced mixing must always be computed simultaneously.
Computations of element settling have been described many times. Here we use the same prescriptions as in Richard et al. (1996) with collision cross sections and microscopic diffusion coefficients as given by Paquette et al. (1986). Radiative accelerations are neglected, as usual in these cool stars (precise computations in the Sun by Turcotte et al. (1998) show that they are indeed negligible for our purpose).

Rotation-induced mixing is also computed as in Richard et al. (1996) except for the introduction of the $\mu$-currents in the velocity of meridional circulation. Following Zahn (1992) and (1993), meridional circulation is suppose to induce shear flow instabilities which, in a density stratified medium, lead to large horizontal diffusivities. The combination of the circulation and horizontal mixing may be treated as a vertical effective diffusion process, described with an effective diffusion coefficient:

$D_{\text {eff }} \simeq \frac{1}{C_{\mathrm{h}}} U_{\mathrm{r}} \cdot r \quad$ with $\quad C_{\mathrm{h}} \simeq 30$.

Abundance variations of helium, lithium and heavier elements are computed within this framework, all along evolutionary sequences. The modifications of the internal structure induced by element diffusion are taken into account in the computations, as in Richard et al. (1996).

\subsection{Self-regulating process}

The originality of the present paper lies in the introduction of $\mu$-currents in the meridional circulation. As discussed in Paper I and verified in the complete numerical computations (see below), $\mu$-currents become of the same order as $\Omega$-currents for small $\mu$-gradients, rapidly reached from helium settling below the convective zone. In this case we claim that, under some conditions, a self-regulating process may take place in which both the circulation and the settling are strongly reduced.

At the beginning of the stellar evolution, $\left|E_{\mu}\right|$ is smaller than $\left|E_{\Omega}\right|$; meridional circulation proceeds and induces transport of chemical elements coupled with settling. The helium concentration gradient below the convective zone increases (in absolute value) leading to an increasing vertical $\mu$-gradient. The horizontal $\mu$-gradient $\Lambda$ follows proportionally until $\left|E_{\mu}\right|$ becomes of the same order as $\left|E_{\Omega}\right|$, where the circulation velocity is expected to vanish.

Then the whole process is modified and does not follow any longer the classical treatment of the Eddington-Sweet circulation. Let us call $\Lambda_{\text {crit }}$ the horizontal $\mu$-gradient for which $\left|E_{\mu}\right|=\left|E_{\Omega}\right|$. Without microscopic diffusion, the whole circulation would become frozen, keeping horizontal $\mu$-gradients of the order of $\Lambda_{\text {crit }}$ in every layer. However microscopic diffusion still proceeds below the convective zone, leading to a slight decrease of $\Lambda$ in the upper radiative layers (this is due to the fact that there is no horizontal $\mu$-gradient inside the convective zone because of rapid mixing).

As $\Lambda$ becomes smaller than $\Lambda_{\text {crit }}$, circulation proceeds just below the convective zone, in the direction of $U_{\Omega}$. 
Doing so, due to mass conservation, it lifts up matter from below in an ascending flow and pushes it down in a descending flow. Although this description looks like that of normal meridional circulation, there is a fundamental difference: in the deeper layers, where $\Lambda$ was close to $\Lambda_{\text {crit }}$, this extra motion creates an over-critical horizontal $\mu$-gradient, which leads to a local horizontal motion in the direction of $U_{\mu}$. Then part of the matter which falls down in the descending flow is transferred to the ascending flow and goes back up to the convective zone where it came from. We can infer that, when this occurs, the global element depletion from the convective zone is reduced in a significant way, leading to time scales larger than the stellar ages.

A good treatment of this self-regulating process needs a complete numerical simulation, which is planned for a forthcoming paper. Here we used the following approximation.

In the layers where the circulation time scale (that is, the time scale of both the $\Omega$-currents and the $\mu$-currents taken separately) is smaller than the settling time scale, we assumed that, as soon as $\left|E_{\mu}\right|$ becomes of the same order as $\left|E_{\Omega}\right|$ (within $10 \%$ ), the $\mu$-gradient remains equal to the equilibrium one.

On the contrary, in the layers where the circulation time scale is larger than the settling time scale, we assumed that the readjustement would have no time to take place in a settling time scale and element depletion proceeds unaltered.

In other words, the $\mu$-gradient remains constant below the convective zone, where $U_{\Omega}$ is larger than the settling velocity, as soon as $\left|E_{\mu}\right| \simeq\left|E_{\Omega}\right|$. Meanwhile the overall helium and lithium abundances are still subject to depletion due to diffusion deeper in the star, at the place where the settling velocity becomes larger than $U_{\Omega}$ (see below, Fig. 2).

\section{Computational results}

\subsection{Evolution of $\mu$-currents with time in low metallicity stars}

First we present the results obtained for four low metallicity stars with masses of $0.75 M_{\odot}$ (hot end of the plateau), $0.70 M_{\odot}$ (middle of the plateau), $0.65 M_{\odot}$ (cool end of the plateau) and $0.60 M_{\odot}$ to show the behavior of the $\mu$ currents with time. Here the rotation velocity is assumed constant, equal to $V_{\text {rot }}=5 \mathrm{~km} \mathrm{~s}^{-1}$ and the metallicity is chosen equal to $[\mathrm{Fe} / \mathrm{H}]=-2.0$.

Figures 1a-d display the $\left|E_{\Omega}\right|$ and $\left|E_{\mu}\right|$ profiles below the convective zones at different evolutionary stages. Each star arrives on the main sequence with nearly homogenous composition. At that time, $\left|E_{\mu}\right|$ is smaller than $\left|E_{\Omega}\right|$ everywhere inside the star. Meridional circulation and element settling can take place in the radiative regions of the star as described above. As a consequence of the helium settling, the $\mu$-currents increase below the convective zone and become rapidly of the same order as $\Omega$-currents.

\subsection{Lithium abundance variations in plateau stars}

The lithium abundance variations with time have been computed using the following scenario: microscopic diffusion damped by rotation-induced mixing occurs normally until the relative difference between $\left|E_{\mu}\right|$ and $\left|E_{\Omega}\right|$ becomes smaller than $\cong 10 \%$. Then the self-regulating process is supposed to take place, in which diffusion and mixing are strongly reduced (with time scales larger than the stellar lifetime). This occurs down to the place where the microscopic diffusion velocity becomes preponderant (Figs. 2a-c). Below this layer, diffusion is assumed to proceed freely.

Figure 3 presents the lithium abundance variations with time for a bunch of different rotational velocities in a $0.70 M_{\odot}$ star. For small rotation velocities, the lithium abundance variations are mainly due to microscopic diffusion, decreasing for increasing rotation velocity. For velocities larger than $6 \mathrm{kms}^{-1}$ the depletion is mainly due to nuclear destruction, which now increases with increasing velocity. For velocities between 2.5 and $7 \mathrm{~km} \mathrm{~s}^{-1}$, the resulting dispersion is of order 0.06 dex, going up to .12 dex if the $7.5 \mathrm{~km} \mathrm{~s}^{-1}$ possibility is included.

Figures $4 \mathrm{a}-\mathrm{c}$ present the lithium abundance variations with time in the outer layers of the four low-metallicity stars $([\mathrm{Fe} / \mathrm{H}]=-2)$ with three different rotation velocities: $2.5,5.0$ and $7.5 \mathrm{~km} \mathrm{~s}^{-1}$. The $0.65 M_{\odot}$ and $0.70 M_{\odot}$ stars show very similar abundances and their variations with the rotation velocity is small except for the largest value of $7.5 \mathrm{~km} \mathrm{~s}^{-1}$. In the $0.75 M_{\odot}$ star the lithium abundance is more sensitive to the rotation velocity due to the smaller diffusion time scale. For small velocities the lithium value in the $0.75 M_{\odot}$ star is smaller than in cooler stars while it is larger for velocities larger than $5 \mathrm{~km} \mathrm{~s}^{-1}$. For the cooler $0.60 M_{\odot}$ star, lithium is always more depleted due to the small distance between the bottom of the convective zone and the nuclear destruction layer.

Figures 5a-d display, as an example, the abundance profiles for lithium inside the four halo stars, for a rotation velocity of $6 \mathrm{kms}^{-1}$.

\subsection{Influence of metallicity and mixing length parameter on the lithium abundances}

We have tested the influence of metallicity on the lithium abundance variations for all the stars, with two different metallicities $[\mathrm{Fe} / \mathrm{H}]=-2.0$ and $[\mathrm{Fe} / \mathrm{H}]=-3.5$. The curves representing the lithium variations with time are plotted in Fig. 6. These curves are very close, which leads to the conclusion of a very small dependence of the lithium abundance on metallicity.

We have also tested the influence of varying the mixing length parameter used for the computation of the convective zone: the results are presented in Fig. 7. The differences are very small: the results are not sensitive to small variations of the depth of the convective zone. 

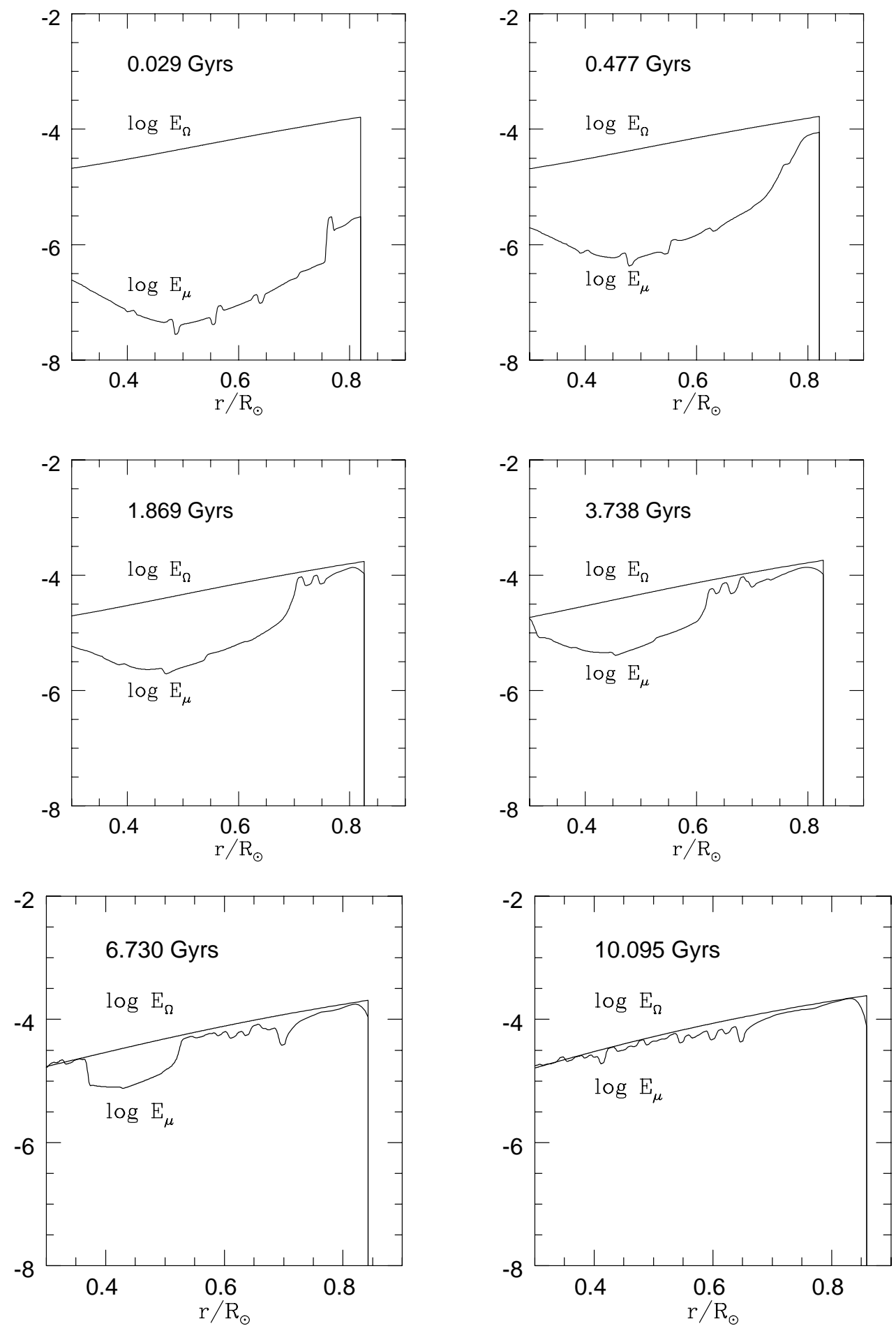

(a)

Fig. 1. a) Evolution of $\mu$-currents with time inside the star. The graphs show the variations with depth of both $\left|E_{\Omega}\right|$ and $\left|E_{\mu}\right|$ (defined in Eqs. (4) and (5)) in a $0.75 M_{\odot}$ halo star with $[\mathrm{Fe} / \mathrm{H}]=-2$ and $V_{\text {rot }}=5 \mathrm{~km} \mathrm{~s}^{-1}$. b) Same figure as Fig. 1a for a $0.70 M_{\odot}$ halo star. c) Same figure as Fig. 1a for a $0.65 M_{\odot}$ halo star. d) Same figure as Fig. 1a for a $0.60 M_{\odot}$ halo star.

\subsection{The case of cool stars: Introduction of a tachocline}

The lithium depletion obtained for the $0.60 M_{\odot}$ star, which is cooler than the lithium plateau, is not sufficient to account for the observations, except for a rotation velocity of $7.5 \mathrm{~km} \mathrm{~s}^{-1}$. The observations show, for these effective temperatures, a lithium depletion by at least a factor of 10 , while the computations obtained by using the same 

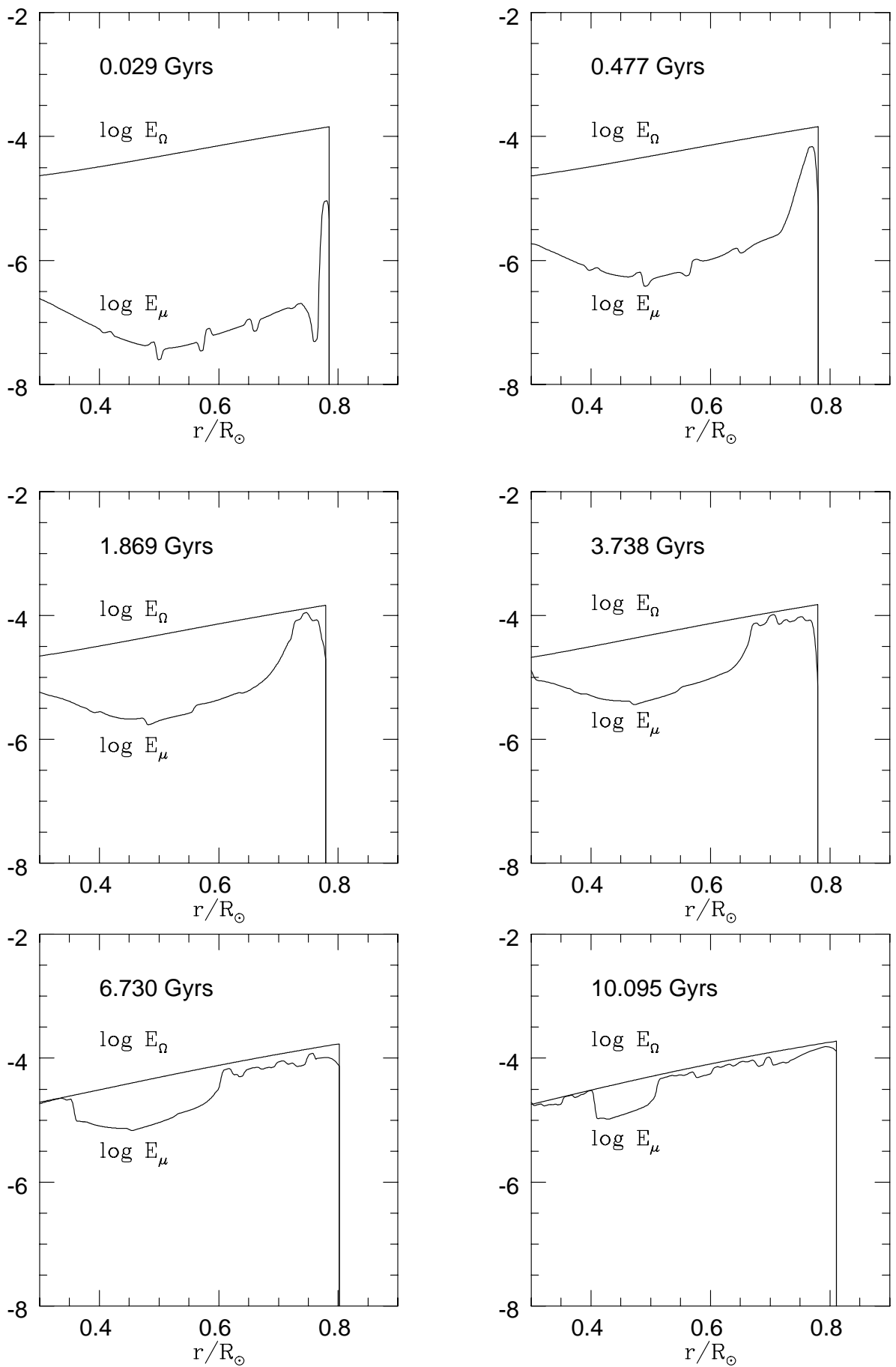

(b)

Fig. 1. continued.

physical inputs as those introduced for the three others stars give a lithium depletion of only $60 \%$ for $2.5 \mathrm{~km} \mathrm{~s}^{-1}$ and $80 \%$ for $5 \mathrm{~km} \mathrm{~s}^{-1}$.

However, at such effective temperatures, a tachocline layer is supposed to take place below the convective zone, as in the Sun (Spiegel \& Zahn 1992). We introduced in the computations an exponential mixing just below the convective region to reproduce the effects of this tachocline. The width of the mixed layer was taken as $0.01 R_{*}$ and the diffusion coefficient was $100 \mathrm{~cm}^{2} \mathrm{~s}^{-1}$ below the convective zone, decreasing exponentially underneath.

We then obtained the lithium variation with time as plotted in Fig. 8 for a velocity of $5 \mathrm{~km} \mathrm{~s}^{-1}$, giving a lithium depletion by a factor 20 , consistent with the observations. We have further checked that such a narrow tachocline layer, if present in hotter stars, would not modify the previously obtained results. 

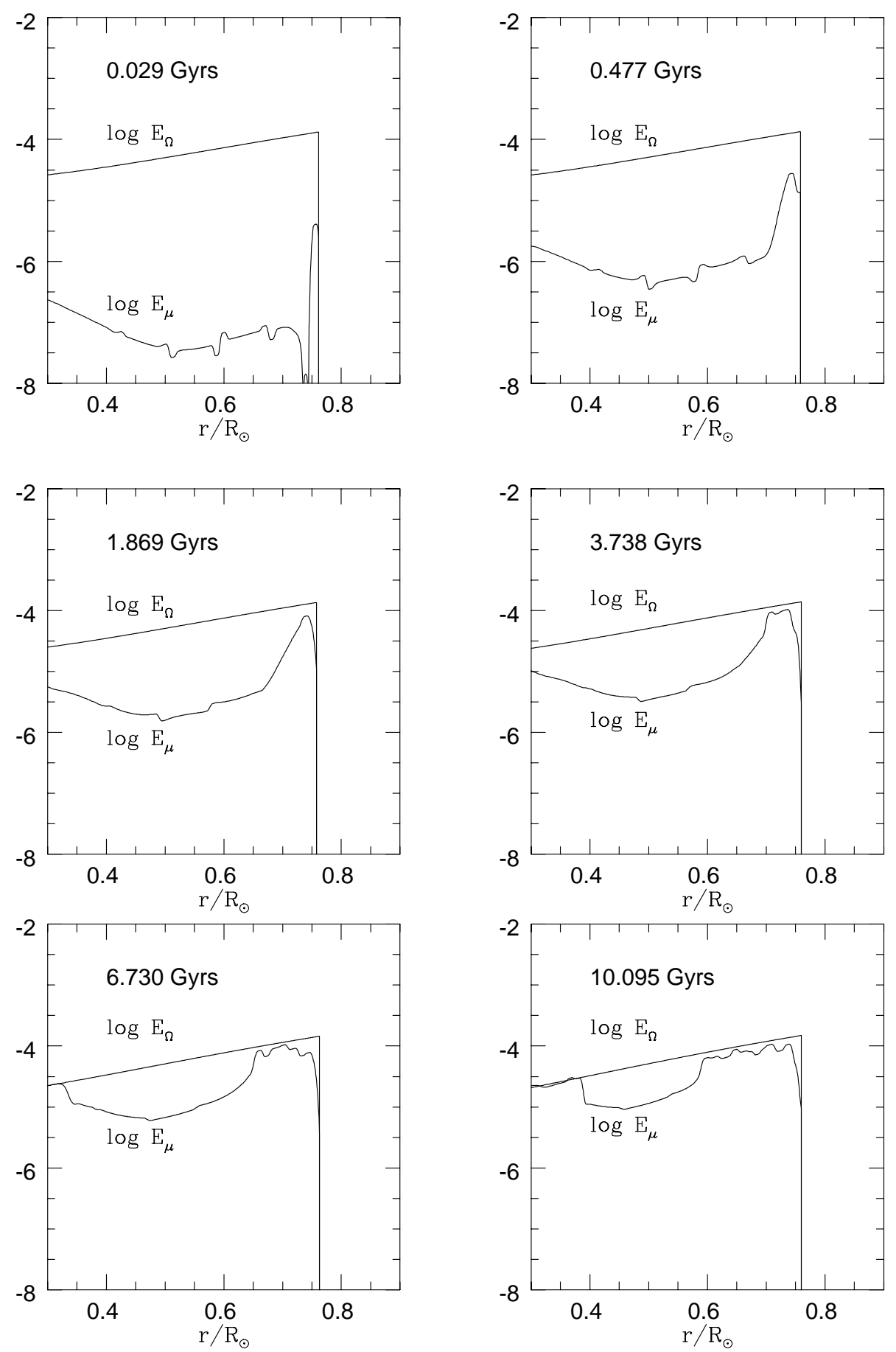

(c)

Fig. 1. continued.

\subsection{Abundance variations of ${ }^{6} \mathrm{Li}$}

We have done the same computations for ${ }^{6} \mathrm{Li}$ as for ${ }^{7} \mathrm{Li}$ in the plateau stars. Figure 9 displays the results for a $0.70 M_{\odot}$ star with metallicity $[\mathrm{Fe} / \mathrm{H}]=-2$ and the same values of the rotation velocity as for ${ }^{7} \mathrm{Li}$ in Fig. 3 . The results differ for the two isotopes because ${ }^{6} \mathrm{Li}$ is more easily destroyed by nuclear reactions than ${ }^{7} \mathrm{Li}$, while the influence of microscopic diffusion is about the same. For small rotation velocities, less than $7 \mathrm{~km} \mathrm{~s}^{-1},{ }^{6} \mathrm{Li}$ is destroyed by

about a factor of two. For larger velocities it goes up to a factor of 5 . In any case, if ${ }^{6} \mathrm{Li}$ is present at the beginning of the stellar evolution, a non negligible amount remains at the age of halo stars.

\section{Discussion}

The results obtained in this paper are compared to the observational points and error bars given by Bonifacio \& Molaro (1997) in Figs. 10a and b. The solid curve drawn 

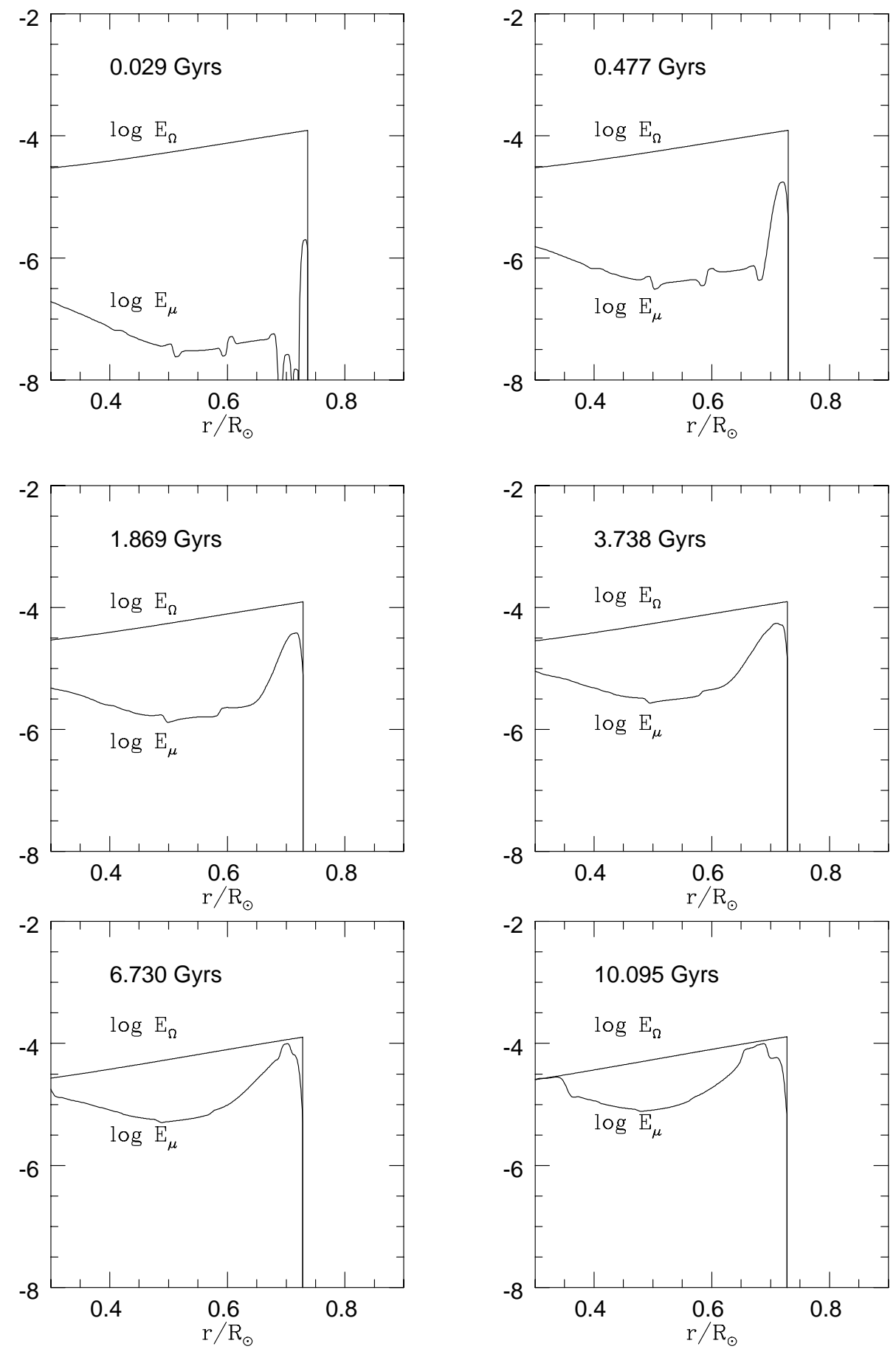

(d)

Fig. 1. continued.

in Fig. 10a represents averaged depletion values for rotation velocities between 2.5 and $7.5 \mathrm{~km} \mathrm{~s}^{-1}$. The original lithium value is $A(\mathrm{Li})=2.5$. In Fig. $10 \mathrm{~b}$, two extreme curves are given, again with the assumption of rotation velocities between 2.5 and $7.5 \mathrm{~km} \mathrm{~s}^{-1}$. The expected dispersion is very small (of order $0.1 \mathrm{dex}$ ) at the middle of the lithium plateau. It becomes larger at both extremes: for the hotter side, it is due to the shorter diffusion time scale and for the cooler side to the proximity of the nuclear destruction layers.

A complete treatment of the self-regulating process described in Sects. 4-3 would need numerical simulations which are in preparation. Here we have assumed that this effect significantly reduces both the circulation and the diffusion as the circulation time scale is smaller than the diffusion time scale while it is supposed to proceed unaltered below. Such a simple scheme gives results consistent with the lithium observations in halo stars. No mass loss has been taken into account, which means that the mass 

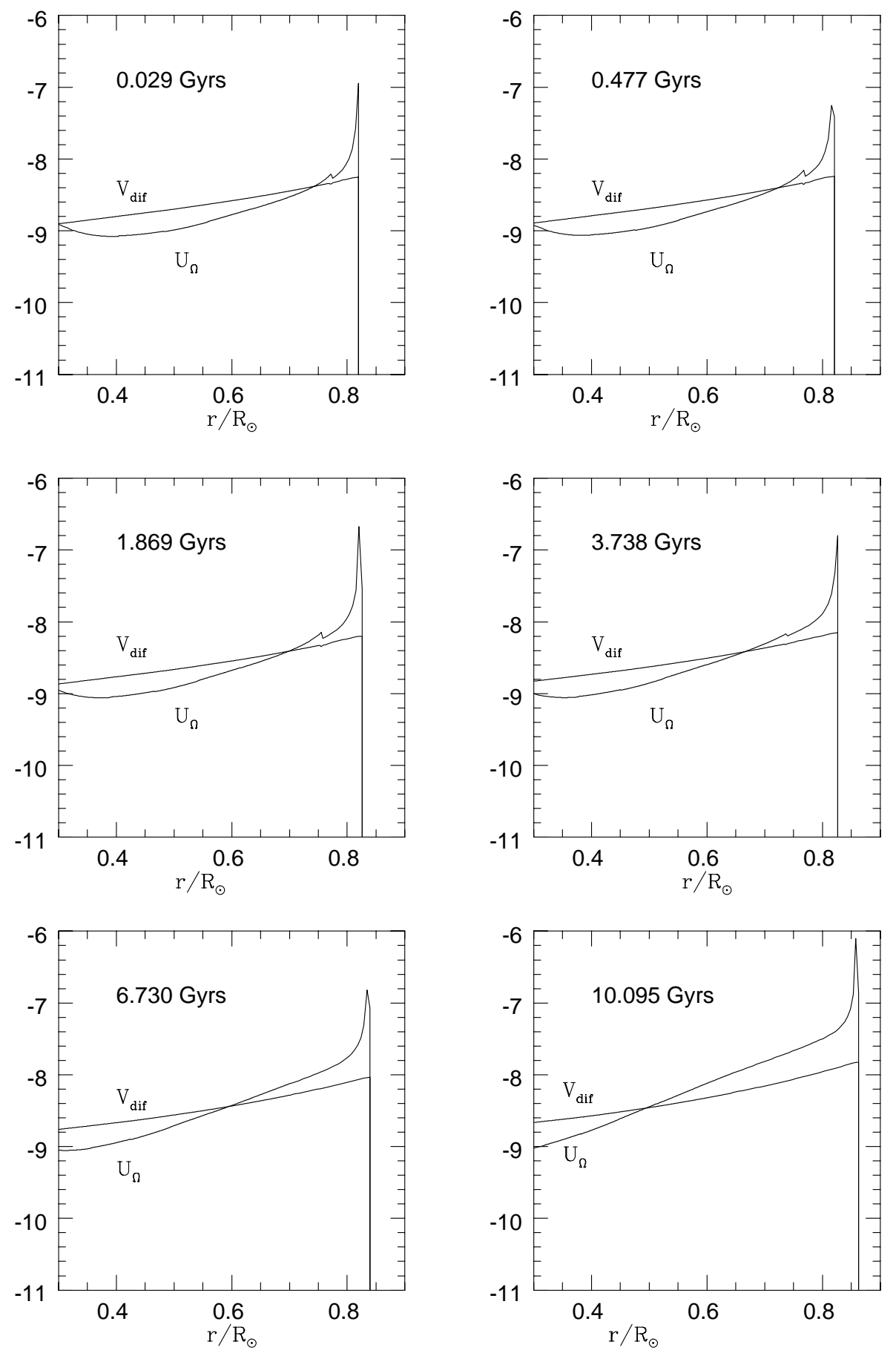

(a)

Fig. 2. a) Microscopic diffusion velocity below the convective zone of a $0.75 M_{\odot}$ star compared to the classical meridional circulation velocity $U_{\Omega}$ (defined in Eq. (10)). When $V_{\text {dif }}<U_{\Omega}$, the self-regulating process can occur while when $V_{\text {dif }}>U_{\Omega}$, the circulation cannot compensate for the effect of diffusion. b) Same figure as Fig. 2a for a $0.70 M_{\odot}$ star. c) Same figure as Fig. 2a for a $0.65 M_{\odot}$ star.

loss rate is assumed smaller than $10^{-13} M_{\odot} \mathrm{yr}^{-1}$ (Vauclair \& Charbonnel 1995).

Two important assumptions have been used in these computations: the stellar rotation velocity was taken as a constant during evolution and the differential rotation inside the stars was assumed negligible.

The first assumption is impossible to check directly, as we have no access to the past rotational history of halo 

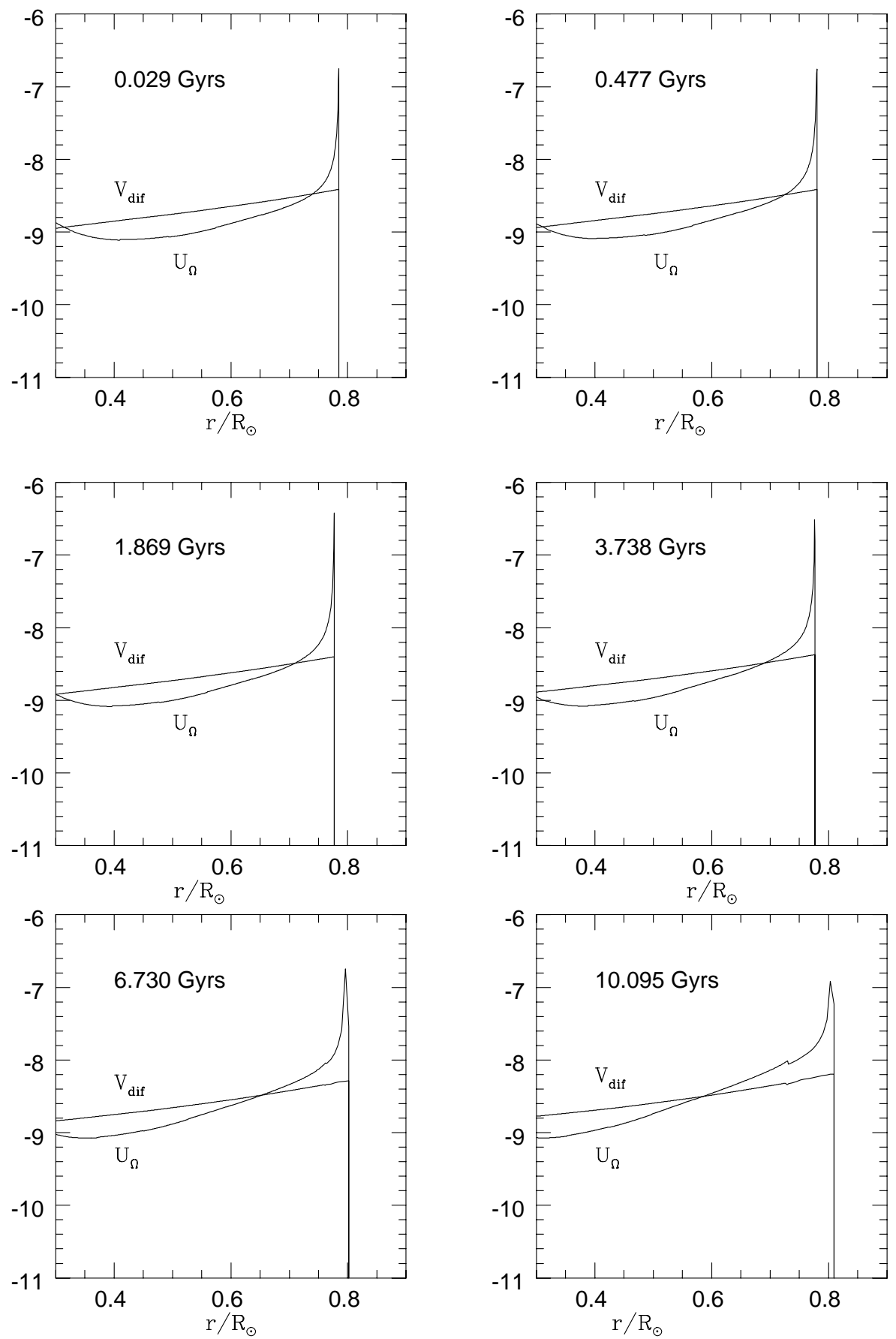

(b)

Fig. 2. continued.

stars. It is generally assumed that they suffer the same rotational breaking as pop I stars. However they have not been formed in the same way, not being in the same galactic sites, and their kinematics is different. There is no reason why halo stars should behave exactly in the same way as open cluster stars. If the rotational velocity of halo stars was larger in the past, we would expect a larger lithium destruction before the self-regulating process we have described takes place. This cannot be excluded, so that the result we give below has to be taken as the lower limit of the primordial value.
The second assumption is supported by recent helioseismic results, which show that the internal rotation of the Sun follows a solid body law except in the convective zone. We may suppose that the same occurs in halo stars. The fact that the obtained results nicely reproduce the observations argue in favour of this hypothesis. Note that the most efficient way to reach this situation seems to be the presence of a very small magnetic field (Mestel et al. 1987; Charbonneau \& MacGregor 1992) which, as discussed by Mestel (1999), would suppress differential 

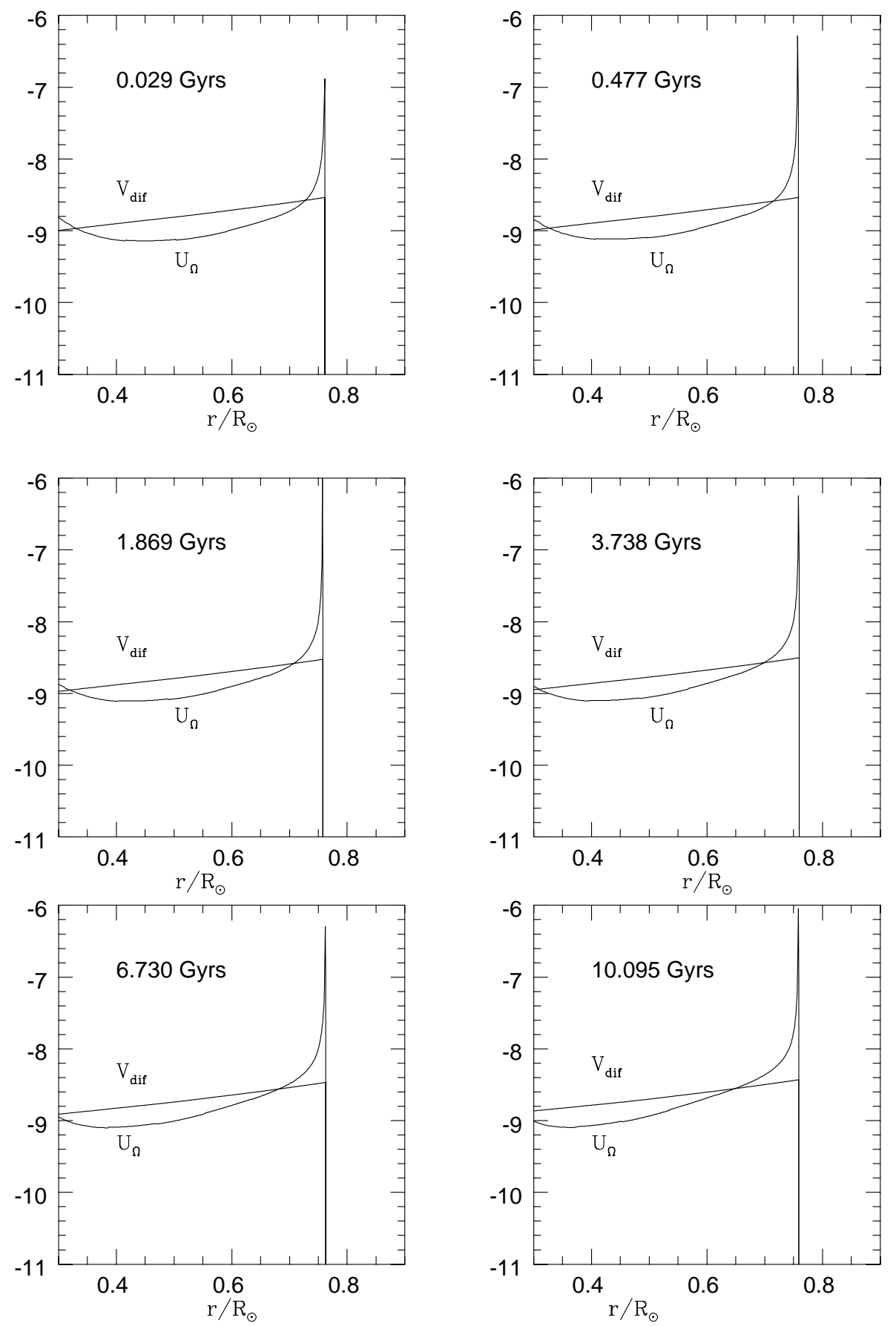

(c)

Fig. 2. continued.

rotation without altering large-scale motion. Such a physics has to be further studied.

It is possible, however, that in some specific stars, due to gravitational interaction with other stars or to some unknown other process, differential rotation does occur. This could explain the existence of some stars with no observed lithium below the plateau: lithium would have been destroyed there by nuclear reactions due to extra mixing. It could also explain why some dispersion is observed in globular cluster stars while it is not observed in field stars (Boesgaard \& Deliyannis 1998).
Futher computations have to be done to describe more precisely the hydrodynamical situation which occurs when the $\mu$-currents oppose the $\Omega$-currents below the convective zones of slowly rotating stars. Applications to lithium in open clusters are underway. The results presented here are very encouraging in this respect. They show that the plateau can be reproduced with no "ad hoc" adjustment of any parameter, with an overall lithium depletion slightly smaller than a factor two. In this case ${ }^{6} \mathrm{Li}$ is still present in the plateau stars. The primordial lithium value is then found as $2.5 \pm 0.1$, consistent with the recent 


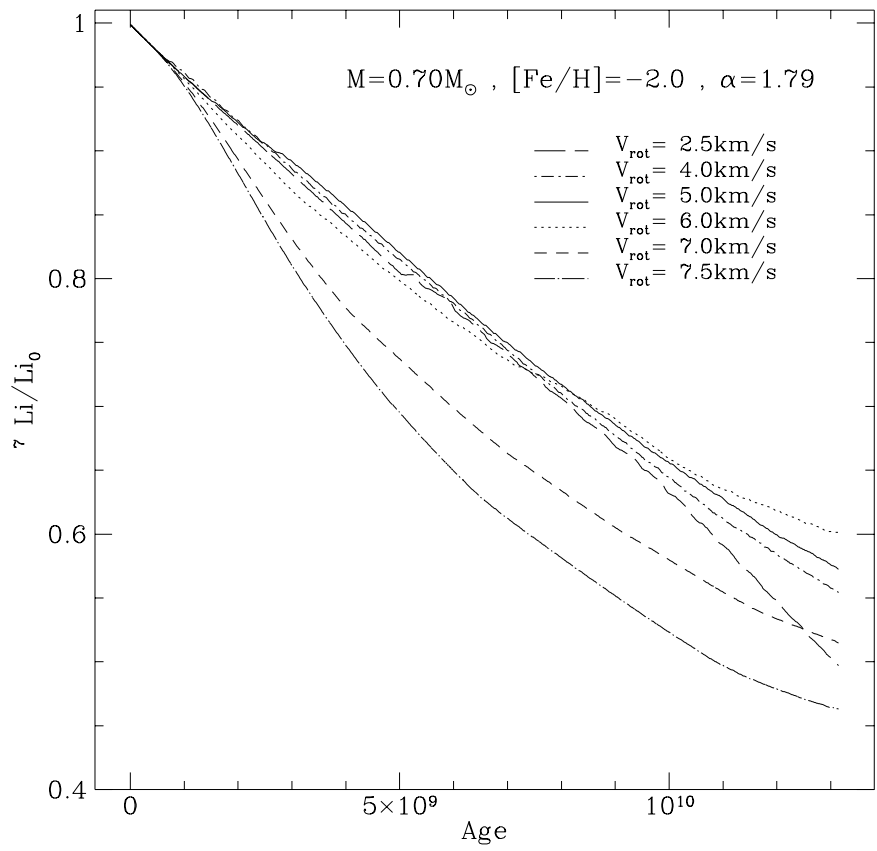

Fig. 3. Lithium abundance variations at the surface of a $0.70 M_{\odot}$ star for rotation velocities between 2.5 and $7.5 \mathrm{~km} \mathrm{~s}^{-1}$. The minimum depletion is obtained for $6 \mathrm{~km} \mathrm{~s}^{-1}$. For smaller velocities, depletion is mainly due to microscopic diffusion while for larger velocities it is mainly due to nuclear destruction. Due to the self-regulating process, the obtained dispersion is quite small.
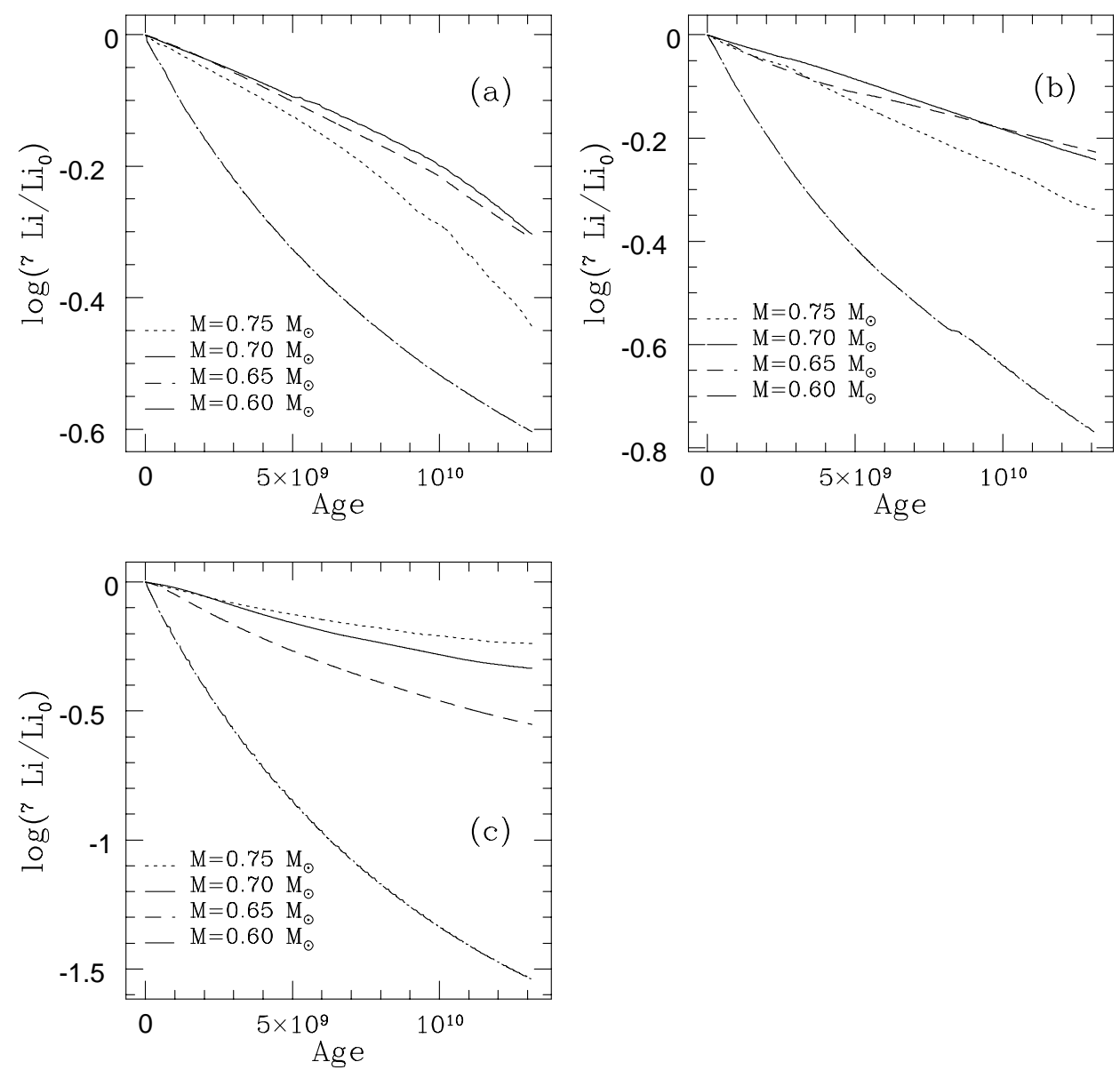

Fig. 4. Lithium abundance variations at the surface of the four halo stars, with three different rotation velocities: a) $2.5 \mathrm{~km} \mathrm{~s}^{-1}$, b) $5 \mathrm{~km} \mathrm{~s}^{-1}$, c) $7.5 \mathrm{~km} \mathrm{~s}^{-1}$. 

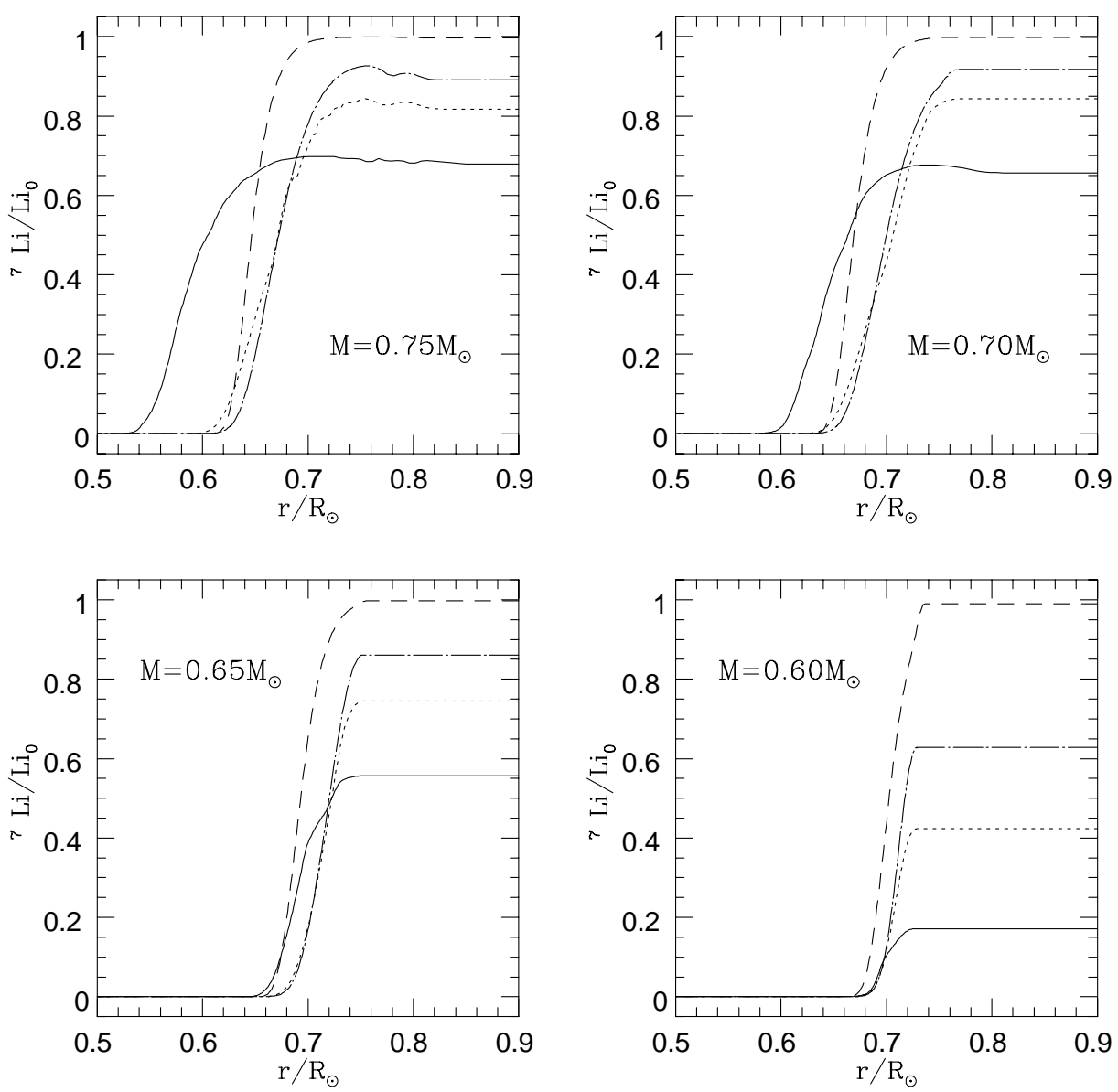

Fig. 5. Lithium profiles inside the four halo stars, with a rotation velocity of $6 \mathrm{~km} \mathrm{~s}^{-1}$. Dashed line: 0.029 Gyrs, long dashed dotted line: 1.869 Gyrs, dotted line: 3.738 Gyrs and solid line: 10.095 Gyrs.

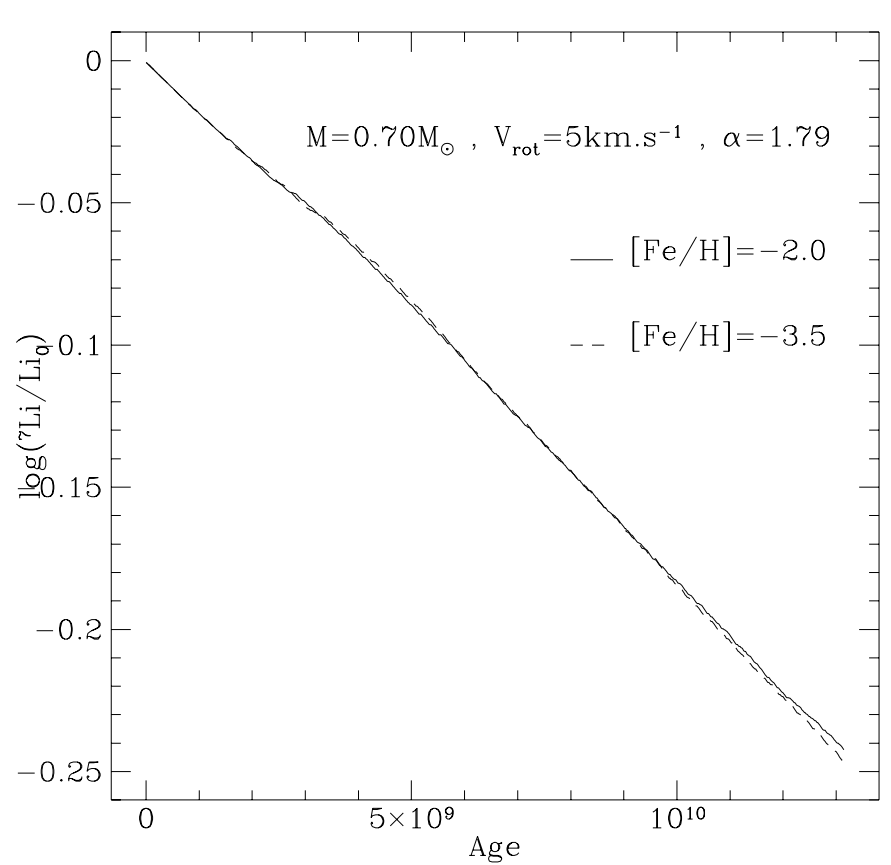

Fig. 6. Lithium abundance variation with time for $0.70 M_{\odot}$ halo stars with two different values of the metallicity.

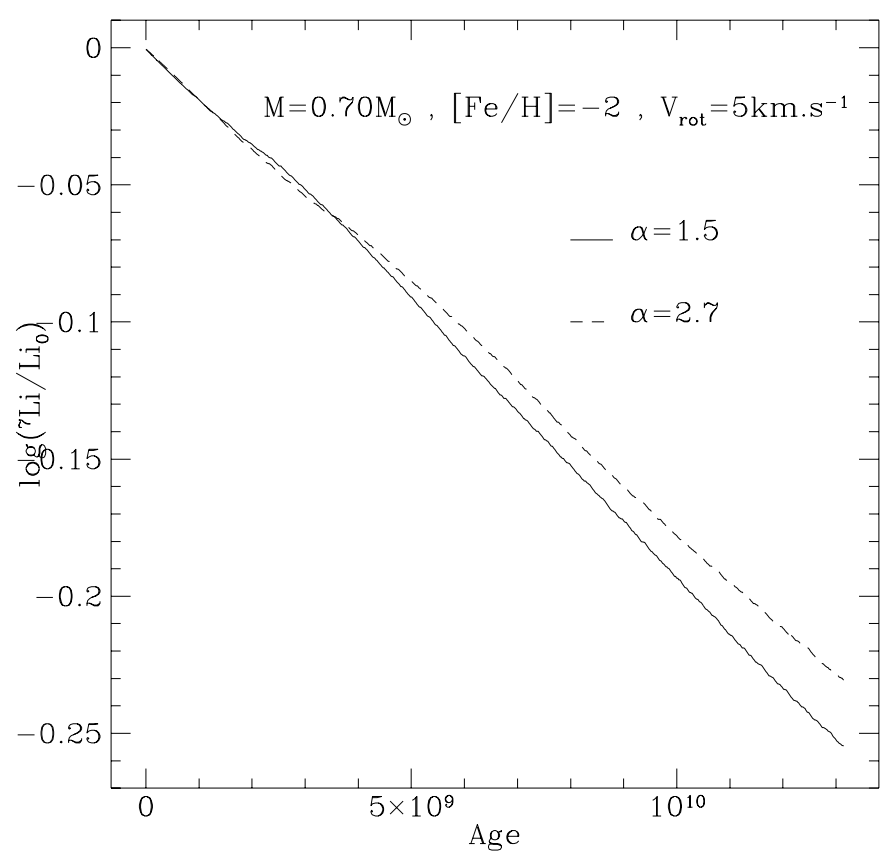

Fig. 7. Lithium abundance variation with time for $0.70 M_{\odot}$ halo stars with two different values of the mixing length parameter. 


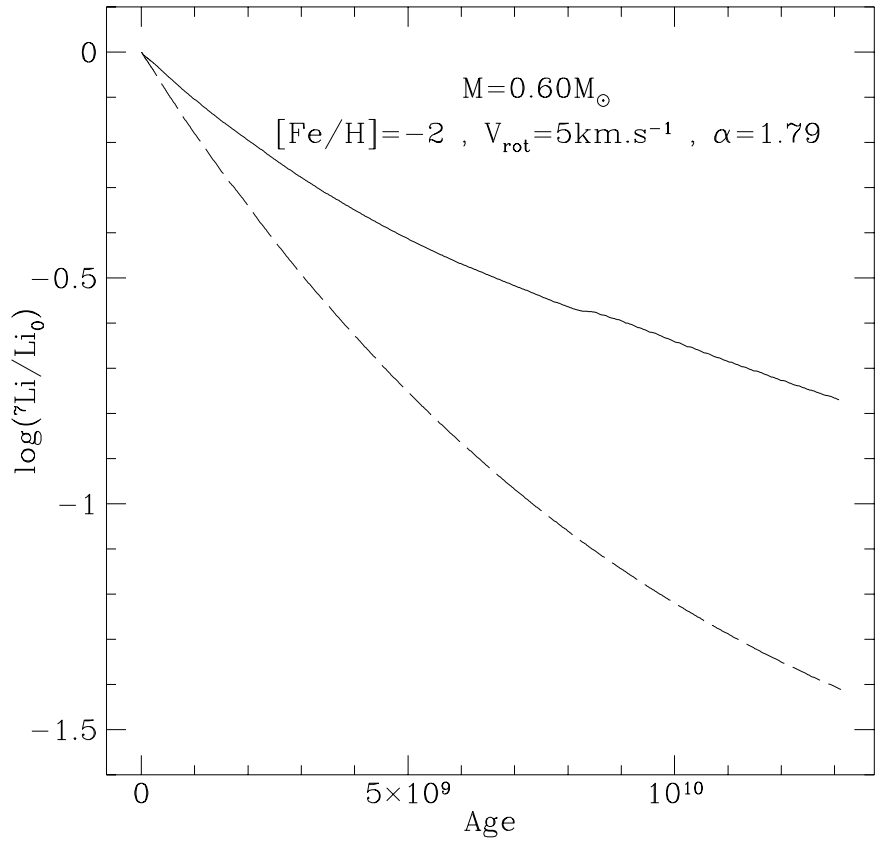

Fig. 8. Lithium abundance variation with time for $0.60 M_{\odot}$ halo stars with and without tachocline. Solid line: stellar model without tachocline, dashed line: stellar model with tachocline.

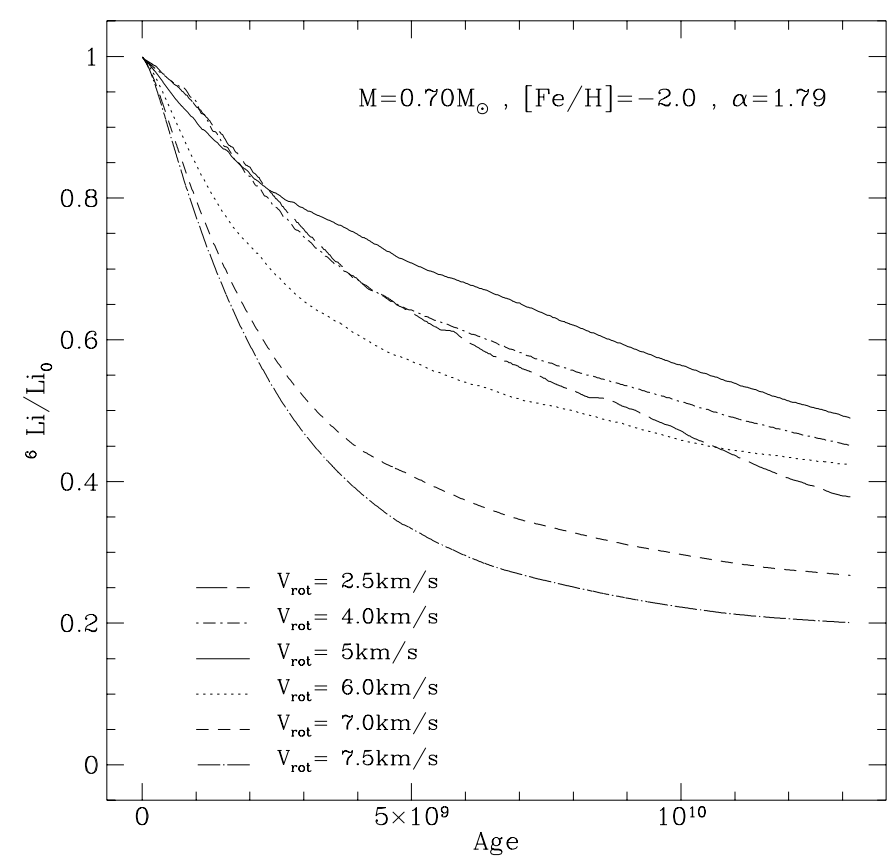

Fig. 9. Lithium 6 abundance variations at the surface of a $0.70 M_{\odot}$ star for rotation velocities between 2.5 and $7.5 \mathrm{~km} \mathrm{~s}^{-1}$.

Table 1. Lithium depletion and effective temperatures of stars for the four considered masses, three different rotation velocities and two ages. Here no tachocline is included in the models.

\begin{tabular}{|c|c|c|c|c|c|c|c|c|c|}
\hline \multicolumn{2}{|l|}{ Masse } & \multicolumn{2}{|c|}{$M=0.75 M_{\odot}$} & \multicolumn{2}{|c|}{$M=0.70 M_{\odot}$} & \multicolumn{2}{|c|}{$M=0.65 M_{\odot}$} & \multicolumn{2}{|c|}{$M=0.60 M_{\odot}$} \\
\hline & & $\log \frac{\mathrm{Li}}{\mathrm{Li}_{0}}$ & $T_{\text {eff }}$ & $\log \frac{\mathrm{Li}}{\mathrm{Li}_{0}}$ & $T_{\text {eff }}$ & $\log \frac{\mathrm{Li}}{\mathrm{Li}_{0}}$ & $T_{\text {eff }}$ & $\log \frac{\mathrm{Li}}{\mathrm{Li}_{0}}$ & $T_{\mathrm{eff}}$ \\
\hline \multirow[t]{2}{*}{$V=2.5 \mathrm{~km} \mathrm{~s}^{-1}$} & 10 Gyrs & -0.29 & 6393 & -0.20 & 6059 & -0.22 & 5718 & -0.52 & 5383 \\
\hline & 12 Gyrs & -0.38 & 6524 & -0.26 & 6139 & -0.28 & 5773 & -0.57 & 5421 \\
\hline \multirow[t]{2}{*}{$V=5.0 \mathrm{~km} \mathrm{~s}^{-1}$} & 10 Gyrs & -0.26 & 6391 & -0.18 & 6059 & -0.18 & 5715 & -0.64 & 5185 \\
\hline & 12 Gyrs & -0.31 & 6473 & -0.22 & 6133 & -0.21 & 5770 & -0.73 & 5226 \\
\hline \multirow[t]{2}{*}{$V=7.5 \mathrm{~km} \mathrm{~s}^{-1}$} & 10 Gyrs & -0.21 & 6397 & -0.28 & 6059 & -0.46 & 5720 & -1.34 & 5385 \\
\hline & 12 Gyrs & -0.23 & 6507 & -0.32 & 6136 & -0.52 & 5774 & -1.47 & 5423 \\
\hline
\end{tabular}



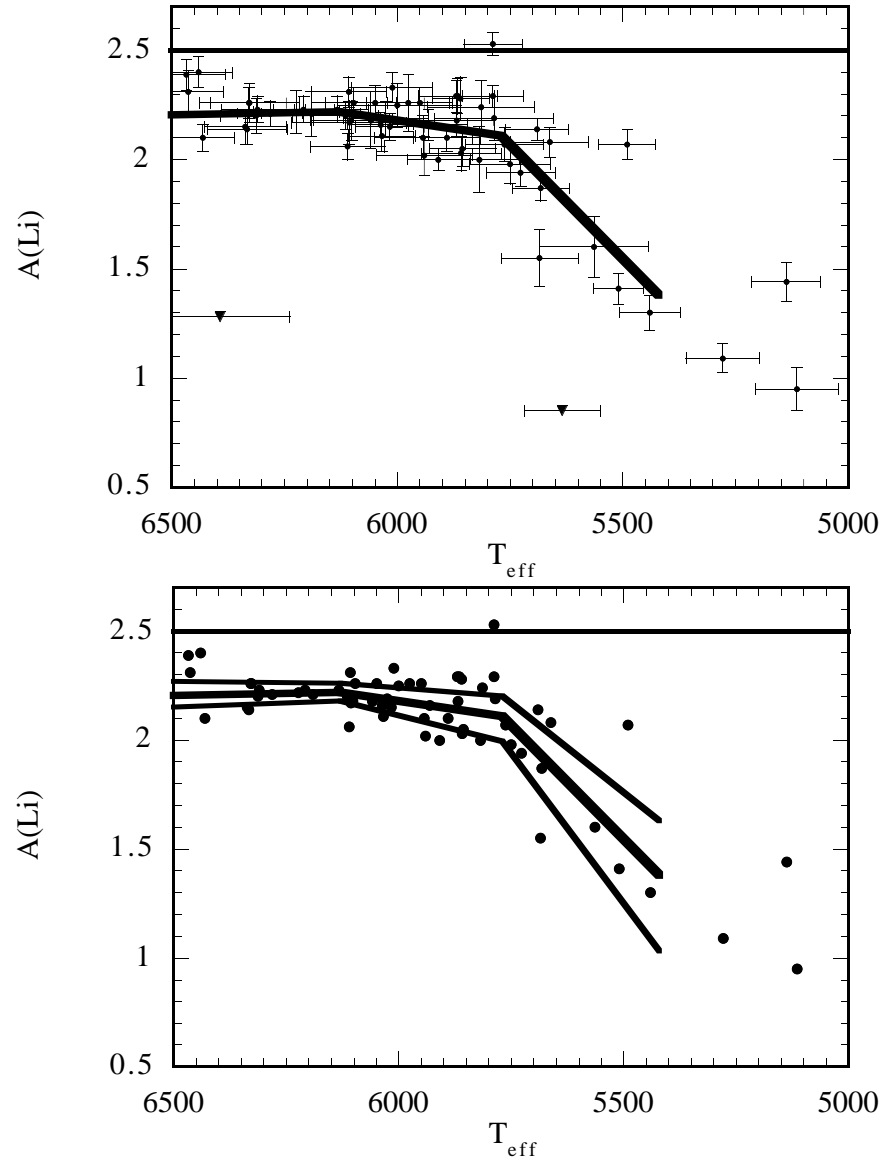

Fig. 10. Comparison between the computations of lithium depletion and the observational values of Bonifacio \& Molaro (1997). a) Average curve obtained for rotation velocities between 2.5 and $7.5 \mathrm{~km} \mathrm{~s}^{-1}$. Here a tachocline is introduced as explained in Sect. 5.4. The original lithium value is choosen as 2.5 to fit the observations. b) Expected dispersion if the rotation velocities lie between 2.5 and $7.5 \mathrm{~km} \mathrm{~s}^{-1}$. The observational error bars are not shown for clarity.

determinations of $\mathrm{D} / \mathrm{H}$ and ${ }^{4} \mathrm{He} / \mathrm{H}$ (see Burles \& Tytler 1998). These results give a baryonic number $\eta=(5 \pm$ 1) $\times 10^{-10}$ and a baryonic density $\Omega_{\mathrm{b}} h^{2}$ between 0.015 and 0.022 .

\section{References}

Alonso, A., Arribas, S., \& Martinez-Roger, C. 1996, A\&AS, 117,227

Blackwell, D. E., Petford, A. D., Haddock, D. J., Arribas, S., \& Selby, M. J. 1990, A\&A, 232, 396

Boesgaard, A. M., Deliyannis, C. P., Stephens, A., \& King, J. R. 1998, ApJ, 493, 206

Bonifacio, P., \& Molaro, P. 1997, MNRAS, 285, 847

Burles, S., \& Tytler, D. 1998, ApJ, 507, 732

Chaboyer, B., \& Demarque, P. 1994, ApJ, 433, 510

Chaboyer, B., Demarque, P., \& Pinsonneault, M. H. 1995, ApJ, 441,865

Charbonneau, P., \& MacGregor, K. B. 1992, ApJ, 387, 639

Deliyannis, C. P., Boesgaard, A. M., \& King, J. R. 1995, ApJ, 452, L13
Deliyannis, C. P., \& Demarque, P. 1991, ApJ, 379, 216

Deliyannis, C. P., Demarque, P., \& Kawaler, S. D. 1990, ApJS, 73,21

Deliyannis, C. P., Pinsonneault, M. H., \& Duncan, D. K. 1993, ApJ, 414, 740

Hobbs, L. M., \& Thorburn, J. A. 1994, ApJ, 428, L25

Hobbs, L. M., \& Thorburn, J. A. 1997, ApJ, 491, 772

Hobbs, L. M., Thorburn, J. A., \& Welty, D. E. 1991, ApJ, 373, L47

Maeder, A., \& Zahn, J.-P. 1998, A\&A, 334, 1000

Mestel, L. 1953, MNRAS, 113, 716

Mestel, L. 1999, Stellar Magnetism (Oxford Science Publications)

Mestel, L., \& Moss, D. L. 1986, MNRAS, 221, 25

Mestel, L., Tayler, R. J., Moss, D. L., 1987, in The Internal Solar Angular Velocity, ed. B. R. Durney, \& S. Sofia (Reidel Pub. Company)

Michaud, G. 1970, ApJ, 160, 641

Michaud, G., Charland, Y., Vauclair, S., \& Vauclair, G. 1976, ApJ, 210, 447

Michaud, G., Fontaine, G., \& Beaudet, G., 1984, ApJ, 282, 206

Molaro, P., Primas, F., \& Bonifacio, P. 1995, A\&A, 295, L47

Norris, J. E., Ryan, S. G., Beers, T. C., \& Deliyannis, C. P. 1997, ApJ, 485, 370

Norris, J. E., Ryan, S. G., \& Stringfellow, G. S. 1994, ApJ, 423,386

Paquette, C., Pelletier, C., Fontaine, G., \& Michaud, G. 1986, ApJS, 61, 177

Pinsonneault, M. H., Deliyannis, C. P., \& Demarque, P. 1992, ApJS, 78, 179

Pinsonneault, M. H., Walker, T. P., Steigman, G., \& Narayanan, V. K. 1999, ApJ, 527, 180

Proffitt, C. R., \& Michaud, G. 1991, ApJ, 371, 584

Richard, O., Vauclair, S., Charbonnel, C., \& Dziembowski, W. A. 1996, A\&A, 312, 1000

Ryan, S. G., Beers, T. C., Deliyannis, C. P., \& Thorburn, J. A. 1996, ApJ, 458, 543

Ryan, S. G., Norris, J. E., \& Beers, T. C. 1999, ApJ, 523, 654

Smith, V. V., Lambert, D. L., \& Nissen, P. E. 1993, ApJ, 408, 262

Smith, V. V., Lambert, D. L., \& Nissen, P. E. 1998, ApJ, 506, 405

Spiegel, E. A., \& Zahn, J.-P. 1992, A\&A, 265, 106

Spite, F., \& Spite, M. 1982, A\&A, 115, 357

Spite, M., Francois, P., Nissen, P. E., \& Spite, F. 1996, A\&A, 307,172

Thorburn, J. A. 1994, ApJ, 421, 318

Turcotte, S., Richer, J., Michaud, G., Iglesias, C. A., \& Rogers, F. J. 1998, ApJ, 504, 539

Vauclair, S. 1988, ApJ, 335, 971

Vauclair, S. 1999, A\&A, 351, 973

Vauclair, S., \& Charbonnel, C. 1995, A\&A, 295, 715

Vauclair, S., \& Charbonnel, C. 1998, ApJ, 502, 372

Vauclair, S., \& Vauclair, G. 1982, ARA\&A, 20, 37

Vauclair, G., Vauclair, S., \& Michaud, G. 1978a, ApJ, 223, 920

Vauclair, S., Vauclair, G., Schatzman, E., \& Michaud, G. 1978b, ApJ, 223, 567

Zahn, J.-P. 1992, A\&A, 265, 115

Zahn, J.-P. 1993, in Astrophysical Fluid Dynamics, ed. J.-P. Zahn, \& J. Zinn-Justin, Les Houches, Session XLVII, 561 